\title{
Influence of laser power in nanosecond laser texturing for a hydrophobic state on SS316L
}

\author{
M. H. Zul'1 , M. Ishak ${ }^{1}$, M. H. Aiman ${ }^{1}$ and M. M. Quazi ${ }^{1}$ \\ ${ }^{1}$ Faculty of Mechanical and Automotive Engineering Technology, Universiti Malaysia Pahang, 26600 Pekan, Pahang, Malaysia \\ Phone: +6094246235; Fax: +6094242202
}

\begin{abstract}
The use of lasers in surface engineering has recently made significant progress. The hydrophobic surface is commonly studied because of the application in various fields, including vehicles, aerospace, biomedicine, etc. Since these laser methods require many combination parameters, such as laser power $(\mathrm{P})$, frequency $(f)$, scan speed $(v)$ and laser beam diameter $(D)$, the effect of the parameters must therefore be investigated to produce the hydrophobic condition. This research tries to relate the laser power with the morphological properties and contact angle of the SS316L surfaces. Samples are subjected to laser texturing with different laser power settings. The surface is then characterised by surface roughness, and the contact angle is measured according to a specific time interval. The laser power output and energy density function on the surface and contact angle were investigated in these contexts experimentally. Surface roughness was defined and validated to show that the laser parameters' effect is effective and controllable. This study shows that the laser output intensity significantly contributes to regulating surface roughness and the substrate's wetting activity. The $18 \mathrm{~W}$ and $24 \mathrm{~W}$ laser outputs produce a spiked surface with various peaks that cause the surface to become hydrophobic over time because of the air-trap that happens in the valley.
\end{abstract}

ARTICLE HISTORY

Received: $18^{\text {th }}$ Jan. 2021

Revised: $13^{\text {th }}$ July 2021

Accepted: 01 ${ }^{\text {st }}$ Nov. 2021

\section{KEYWORDS}

Nanosecond laser texturing; surface roughness;

SS316L;

contact angle.

\section{INTRODUCTION}

Surface modification has various industrial applications because of the number of surface-dependent material properties such as wettability, resistance to corrosion, adhesion and friction. Laser texturing methods have various benefits such as flexibility, accuracy, chemical-free processing, lack of tool use and minimal impact on bulk materials' properties [1] with low cost and minimum maintanence $[2,3]$ suitable for all types of materials like glass, ceramic, polymer and metals. Many studies on laser-structured metallic surfaces show changes in hydrophilic properties to become hydrophobic over time because of adsorption from the atmosphere. Most industries are more inclined to use more advanced and fast laser technologies such as picosecond and femtosecond lasers, but they are costly and not very economical. Ta et al. suggested using nanosecond lasers as a cheaper alternative to producing hydrophobic surface [4] even the process is slow and involves several runs because of the low removal rate of the material to resolve the issues related to ultrafast laser texturing. As nanosecond laser technology is versatile and simple to shape, many parameters must, of course, need to be optimised to achieve the best machining performance. Laser power [5], pulse duration [6], frequency [7], scan speed, hatching parameters and scan loop [8] are among the laser parameters that are often studied.

This laser texture process is using a laser ablation method. In a pulsed laser ablation process, stainless steel melt to a temperature where the atom gains sufficient energy to enter the gaseous phase because of the laser pulse's energy absorption - the molten materials expelled from the cavity because of vapour and plasma pressure and form surface debris. At the end of a pulse, the heat quickly dissipates through the bulk of the substance and the recasting layer is formed. Laser power is a vital process fingerprint candidate because it defines the laser fluence that specifically affects microstructures. The relation between the laser energy density, $\mathrm{E}_{\mathrm{d}}$ (fluence) as a function of the average output power $(\mathrm{P})$ and pulse frequency $(f)$ are as follows [9]:

$$
\mathrm{E}_{d}=\frac{4 \mathrm{P}}{f(\pi) \mathrm{D}^{2}}
$$

Static contact angle (SCA) of the surface, which above $90^{\circ}$ is defined as hydrophobic surface, while for extreme SCA such as the surfaces have a higher than $150^{\circ}$ is called superhydrophobic.In contrast, a SCA that is less than $90^{\circ}$ is known as a hydrophilic surface [10]. The superhydrophobic artificial surface produced through the process of surface modification either through coating or surface structuring has received significant attention in recent years because this surface is suitable for many functions and applications such as biomedical anti-microbial [11], food industry, water purification device and system, medical and surgical equipment, anti-biofouling for marine industry [12], corrosion 
resistance [13, 14] and wear reduction [1, 15] for automotive, aerospace, defence industries, manufacturing (micromachining) and implant material. Two critical factors in determining hydrophobicity of the surface are the chemical composition and surface morphology [16]. Metal materials typically have hydrophobicity surfaces by developing a rough surface design like micro-structuring by laser ablation [17], and the combination of surface energy reduction processes, such as chemical molecular reagent deposits $[12,14,18]$. Some researchers have combined laser ablation methods and second step processes, such as Ngo and Chun [19], [20], which speed up the transformation from hydrophilic to superhydrophobic behaviour. Li, et al. [11] has also used a two-stage process for constructing a superhydrophobic surface to prevent replication of E. Coli bacteria (approximately 90.3\%) and S. Aureus (up to 99.6\%), great to increases the product functionality and working life. Using chemical and organic coating also enables superhydrophobic surfaces to be achieved. This surface impact improves the ability of the surface to resist biofouling and corrosion [12, 14].

In this work, single-step laser texturing of the SS316L material will be carried out by varying the laser output power value. The resulting textured surface will be analysed for surface characterisation and changes in contact angle. Finally, the relationship between surface topography and the water contact angle of SS316L material is discussed.

\section{EXPERIMENTAL SETUP}

\section{Material and Preparation}

AISI SS316L austenitic stainless steel has been widely used in many areas, including agriculture and machining, vehicle, marine and shipyard construction, and petroleum refinery because of its excellent corrosion resistance in an aggressive environment. 316L stainless steel as defined in Table 1, was cut into $10 \mathrm{~mm} \times 10 \mathrm{~mm}(2 \mathrm{~mm}$ thick $)$ as a sample and was cleaned by ethanol and dried out in ambient environments before and after nanosecond lasers were textured.

Table 1. AISI 316L chemical composition utilised for experiments (all values in wt\%)

\begin{tabular}{ccccccccc}
\hline $\mathrm{C}$ & $\mathrm{Mn}$ & $\mathrm{Si}$ & $\mathrm{Cr}$ & $\mathrm{Ni}$ & $\mathrm{Mo}$ & $\mathrm{P}$ & $\mathrm{S}$ & $\mathrm{Fe}$ \\
\hline 0.021 & 1.4 & 0.38 & 17 & 10 & 2.1 & 0.041 & 0.005 & Balance \\
\hline
\end{tabular}

\section{Laser Texturing of the Surfaces}

The laser device system setup is shown in Figure 1. The energy laser beam was transported to the beam expander and then supplied to the mirror's galvanometer. The laser beam was focused on f-theta lens by the computer and finally, the pattern of laser scanning is controlled by the galvanometer. The height of the platform can be changed to adjust the focal point distance. The pulse laser device has a Gaussian power density profile, $10 \mathrm{~ns}$ pulse width, $1064 \mathrm{~nm}$ wavelength and $30 \mathrm{~W}$ maximum power. The laser scan was conducted in atmospheric conditions with a laser beam diameter of $\sim 50 \mu \mathrm{m}$ during laser processing. The machining parameter specifications are explained in Table 2.

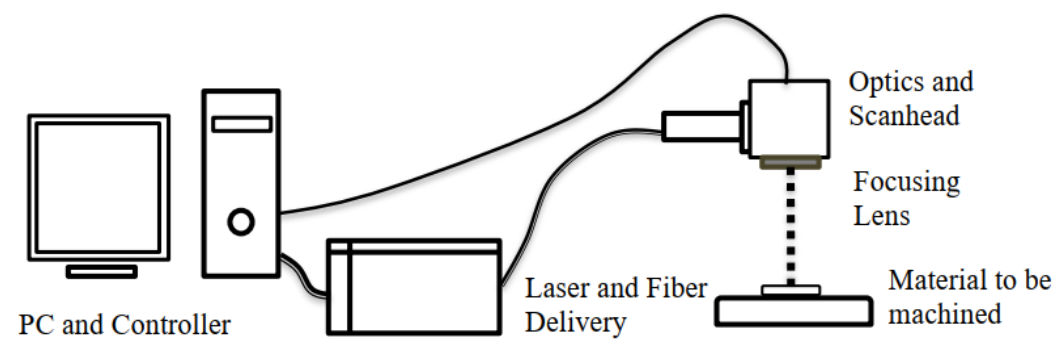

Figure 1. Schematic of laser machining process workstation

Table 2. Laser parameters used in the experiments

\begin{tabular}{cc}
\hline Laser parameters & The values used in the experiments \\
\hline Average Power, $\mathrm{P}$ & $6 \sim 24 \mathrm{~W}$ \\
frequency, $f$ & $20 \mathrm{kHz}$ \\
Scan Speed, $v$ & $20 \mathrm{~mm} / \mathrm{s}$ \\
Laser beam diameter, D & $50 \mu \mathrm{m}$ \\
Pattern & Single line microgroove \\
Hatch line distance & $100 \mu \mathrm{m}$ \\
\hline
\end{tabular}




\section{Surface Characterisation}

Surface topography of the as-received and textured surface was observed using LEXT Olympus OLS5000 3D laser scanning confocal microscope data acquisition application and data analytics application. Standard surface roughness information such as $R_{a}$ (arithmetic average profile roughness), $R_{z}$ (maximum profile height), and $R_{s m}$ (mean profile width) with advanced surface parameters such as $R_{s k}$ (profile skewness) and $R_{k u}$ (profile kurtosis) was calculated with at least 3 measurements taken at different sampling locations. The selected roughness parameters are determined by the formation of solidified material debris on the surface of the textured groove, which, in terms of laser tracer microgeometry, contribute to a decrease in size of textured morphology.

\section{Wettability Experiments}

Contact angle measurements were performed on a custom system device, as shown in Figure 2 for 30 days to determine the hydrophobicity transition by time of textured surfaces at room temperature. The volume of distilled water droplets deposited on the surfaces was controlled through a $0.5 \mu \mathrm{L}$ micropipette. The measurements average is at least three (3) values at various locations. Images were captured by a high-resolution telecentric USB microscope (1.6 x times, adjustable aperture opening). The light source in the backlight offers parallel illumination and increases the image contrast and definition of the object's edges compared to regular lighting, as seen in Figure 2 (a). The sample on the stage with adjustable height is dropped by using micropipette manually with deionised water during measurement as illustrated in Figure 2 (b). The recorded image will be determined by the contact angle via the image-J software using the Drop Analysis plugin.

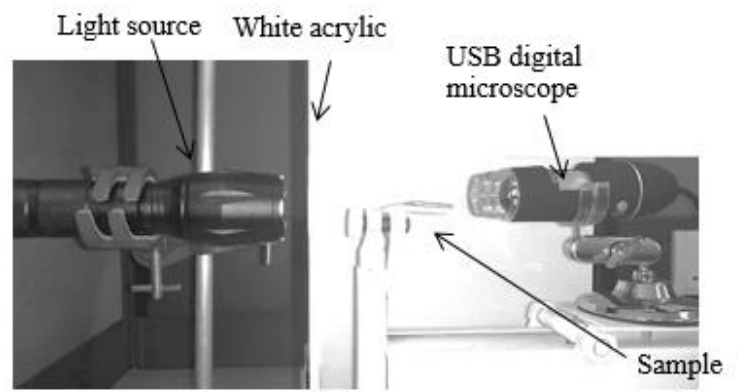

(a)

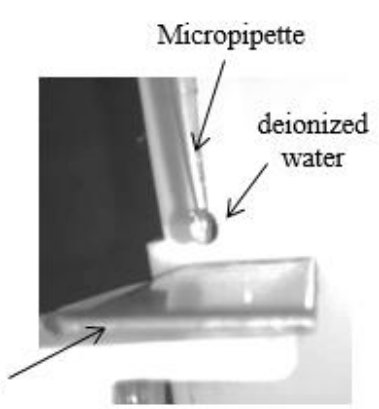

(b)

Figure 2. Experimental setup used for: (a) contact angle meter in this work and (b) water droplet method on the sample

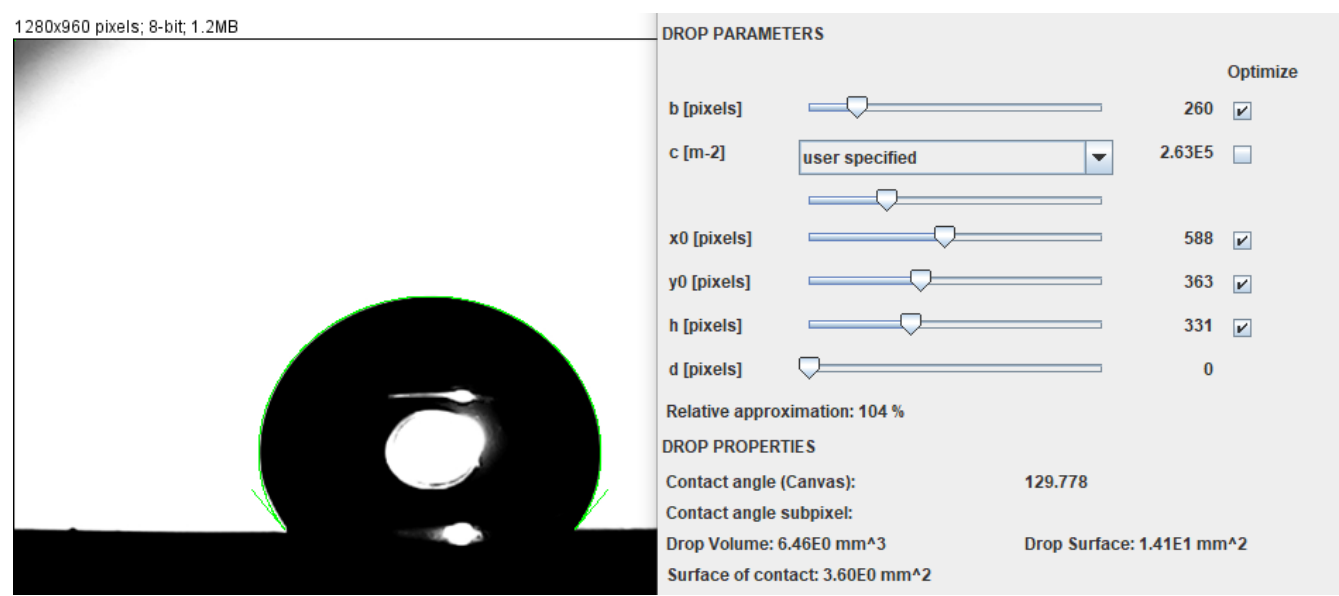

Figure 3. The result from wettability experiment in: (a) contact angle view recorded by USB digital camera and (b) analysis of SCA image via ImageJ Drop Analysis plugin

\section{RESULTS AND DISCUSSION}

\section{Surface Characterization of Textured Surface}

The surface profile of laser-treated surfaces is shown in Figure 3. As observed, all laser-treated surfaces show a horizontal area affected by laser ablation. Laser texturing gives a crater shape obviously to samples with $12 \mathrm{~W}$ and $18 \mathrm{~W}$ compared to $6 \mathrm{~W}$ and $24 \mathrm{~W}$. The laser power used in sample Figure 3 (a) is small and does not significantly affect the material's surface. In contrast, the laser power applied to sample Figure 3 (d) is high, and the effect is almost levelling the entire area. Table 3 displays the $R_{a}$ and $R_{z}$ values for untreated and laser-treated samples from the surface roughness 
profiles. The $R_{a}$ values for the untreated sample is $0.37 \mu \mathrm{m}$, while the $R_{z}$ value is $3.59 \mu \mathrm{m}$. Both the $R_{a}$ and $R_{z}$ values increase considerably after laser treatment, with the $R_{a}$ and $R_{z}$ values ranging averagely from 2.06 to $7.01 \mu \mathrm{m}$ and 33.93 to $43.59 \mu \mathrm{m}$, respectively.

Table 3. The average and standard deviation for surface roughness parameter values extracted from the surface profile

\begin{tabular}{cccccc}
\hline Condition & $\begin{array}{c}\text { Arithmetic } \\
\text { mean, } R_{a}(\mu \mathrm{m})\end{array}$ & $\begin{array}{c}\text { Maximum profile } \\
\text { height, } R_{z}(\mu \mathrm{m})\end{array}$ & $\begin{array}{c}\text { Mean width of } \\
\text { the profile } \\
\text { elements, } R_{s m} \\
(\mu \mathrm{m})\end{array}$ & $\begin{array}{c}\text { Surface } \\
\text { skewness, } R_{s k} \\
(\mu \mathrm{m})\end{array}$ & $\begin{array}{c}\text { Surface kurtosis, } \\
R_{k u}(\mu \mathrm{m})\end{array}$ \\
\hline untreated & 0.37 & 3.519 & 7.753 & -0.05 & 3.66 \\
6W & $2.06 \pm 0.67$ & $33.93 \pm 8.88$ & $40.23 \pm 7.33$ & $-1.86 \pm 0.09$ & $11.26 \pm 2.33$ \\
$12 \mathrm{~W}$ & $5.57 \pm 0.45$ & $34.78 \pm 5.39$ & $54.71 \pm 7.48$ & $1.40 \pm 0.19$ & $3.80 \pm 0.77$ \\
$18 \mathrm{~W}$ & $7.01 \pm 0.48$ & $42.09 \pm 4.27$ & $61.32 \pm 12.78$ & $1.08 \pm 0.21$ & $3.95 \pm 0.85$ \\
$24 \mathrm{~W}$ & $5.54 \pm 0.65$ & $43.59 \pm 6.11$ & $39.17 \pm 1.91$ & $1.71 \pm 0.22$ & $6.07 \pm 0.68$ \\
\hline
\end{tabular}

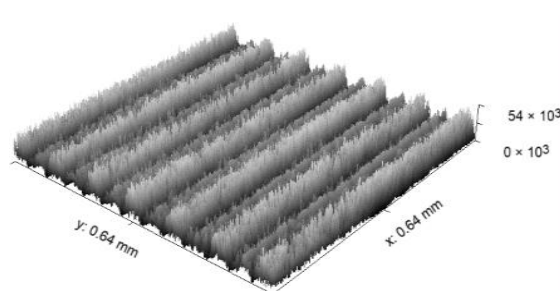

(a)

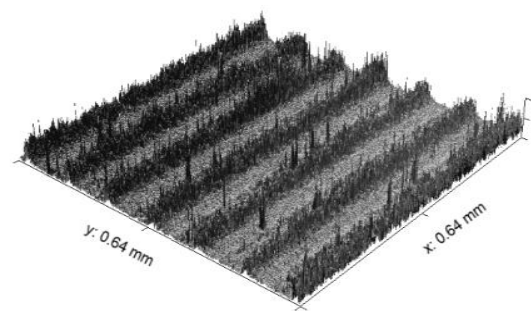

(b)

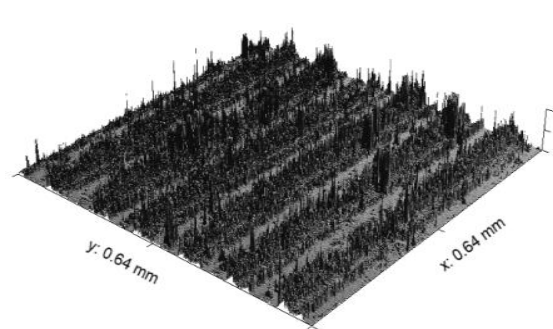

(c)

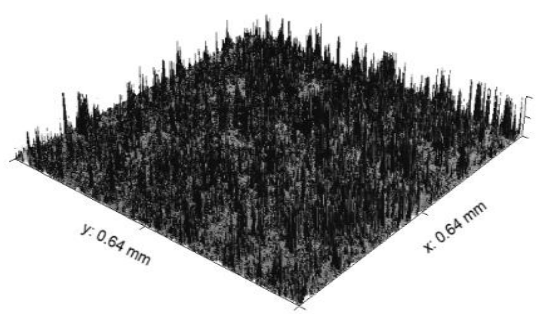

(d)

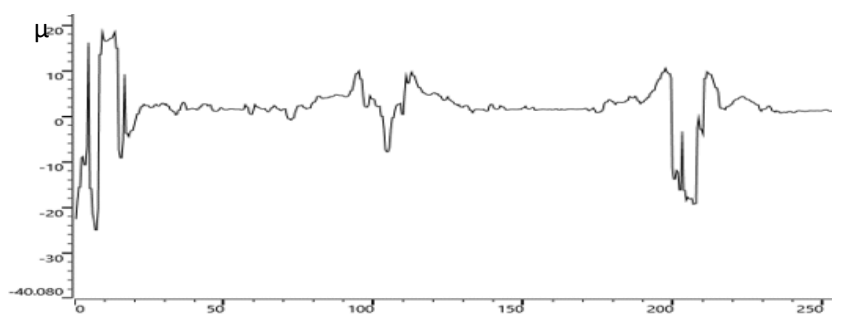

(e)

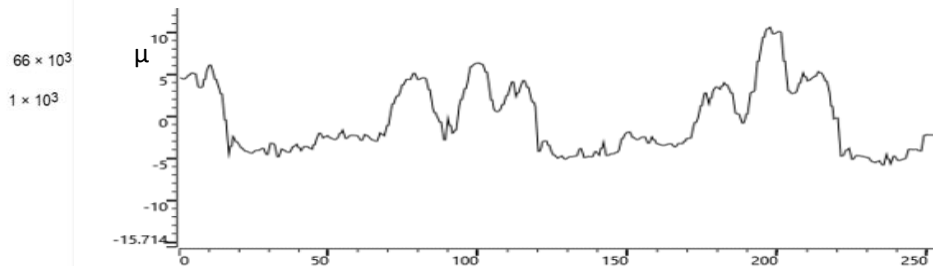

(f)

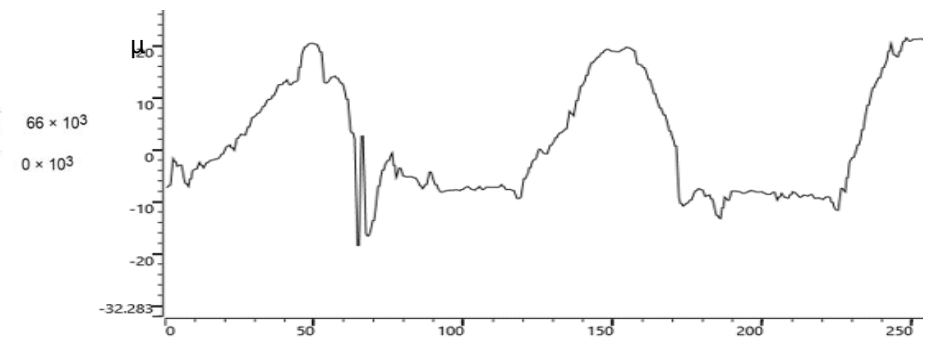

(g)

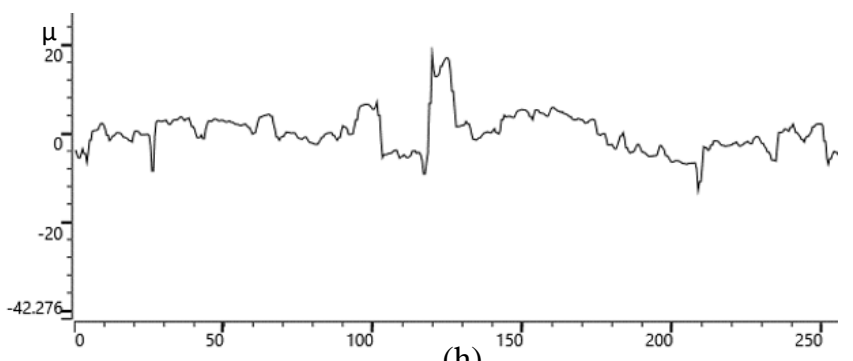

(h)

Figure 4. Laser texturing creates a crater that becomes clearer with increased laser power of: (a d) $6 \mathrm{~W}, 12 \mathrm{~W}, 18 \mathrm{~W}$ and $24 \mathrm{~W}$, respectively. All samples' height profile is shown in $(\mathrm{d} \sim \mathrm{h})$ with the different crater profile with the same laser beam diameter 
The additional (secondary) surface parameters were quantified by $\mathrm{R}_{s k}$ and $\mathrm{R}_{k u}$. These two parameters play an important role in providing a characterisation to the profile contrast between the spikes and valley. Skewness serves to determine whether the surface profile is much against the spikes $\left(\mathrm{R}_{s k}>0\right)$ or valley $\left(\mathrm{R}_{s k}<0\right)$. In contrast, kurtosis will determine whether the surface of the material is flat $\left(R_{k u}<3\right)$ or peak $\left(R_{k u}>3\right)$ [21].

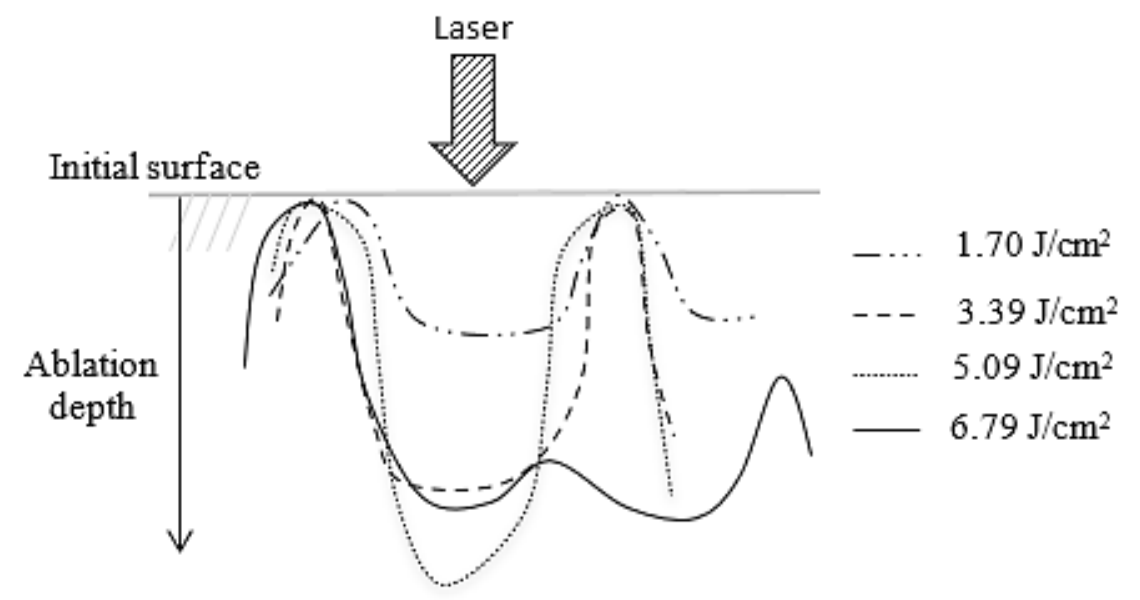

Figure 5. The effect of laser energy on surface topography. The value of peak-to-peak (between crater) is consistently $50 \mu \mathrm{m}$ as set for laser beam diameter

Table 3 shows that the untreated SS316L have a negative $R_{s k}$ value and all samples show the same of $\mathrm{R}_{k u}$ value (>3). In comparison, the $R_{s k}$ value will increase as the laser power increases. As for the $R_{k u}$ parameter, it is the same as $R_{s k}$ except for the smaller laser energy. This also shows that the small value of laser power $(6 \mathrm{~W})$ does not significantly affect laser energy to produce a rough surface, as shown in Figure 4. The depth of laser texturing results in Table 4 also shows that laser power of 12 and $24 \mathrm{~W}$ give depths of 22 and $24 \mu \mathrm{m}$, equal to Ra 5.57 and $5.54 \mu \mathrm{m}$, respectively. By referring to Table 3, the skewness and kurtosis show that untreated SS316L has a bumpy base surface. In contrast, the laser-textured surface shows the valley effect and gives a positive reading of skewness, i.e. the surface has larger peaks amplitude as compared to peak frequency.

Table 4. The ablation depth in laser fluence

\begin{tabular}{ccccc}
\hline $\begin{array}{c}\text { Laser fluence, } \mathrm{J} / \mathrm{cm}^{2} \\
\text { (Power) }\end{array}$ & $\begin{array}{c}1.70 \\
(6 \mathrm{~W})\end{array}$ & $\begin{array}{c}3.39 \\
(12 \mathrm{~W})\end{array}$ & $\begin{array}{c}5.09 \\
(18 \mathrm{~W})\end{array}$ & $\begin{array}{c}6.79 \\
(24 \mathrm{~W})\end{array}$ \\
\hline Ablation depth, $\mu \mathrm{m}$ & 10 & 22 & 28 & 24 \\
\hline
\end{tabular}

\section{Surface Characterization of Textured Surface}

After fabrication, the sample show hydrophilic surface properties with a slight contact angle $\left(<10^{\circ}\right)[22]$ relative to $\sim 70^{\circ}$ of the as-received surface [23]. The rough surface triggered by laser improved these surfaces' wetting behaviour in compliance with Wenzel's model [10]. However, the change of wettability over time is because of modifications in surface chemistry and decomposition of carbon dioxide into carbon with active magnetite [10, 22]. Figure 5 presents the water contact angles of untreated and laser-treated samples. Material surfaces are identified as hydrophobic when the water contact angle is greater than $90^{\circ}[10]$. From the results, untreated samples have a contact angle of $\sim 50^{\circ}$, suggesting the hydrophilic condition at all times. On the other hand, laser-treated water contact angles are found to improve from hydrophilic at day-1 to be hydrophobicity $\left(\sim 125^{\circ}\right)$ at the end of 30 days. This shows that the surface hydrophilicity of SS316L is much improved after laser texturing. Furthermore, the increase in the contact angle is more profound for lasertreated with higher laser power. 


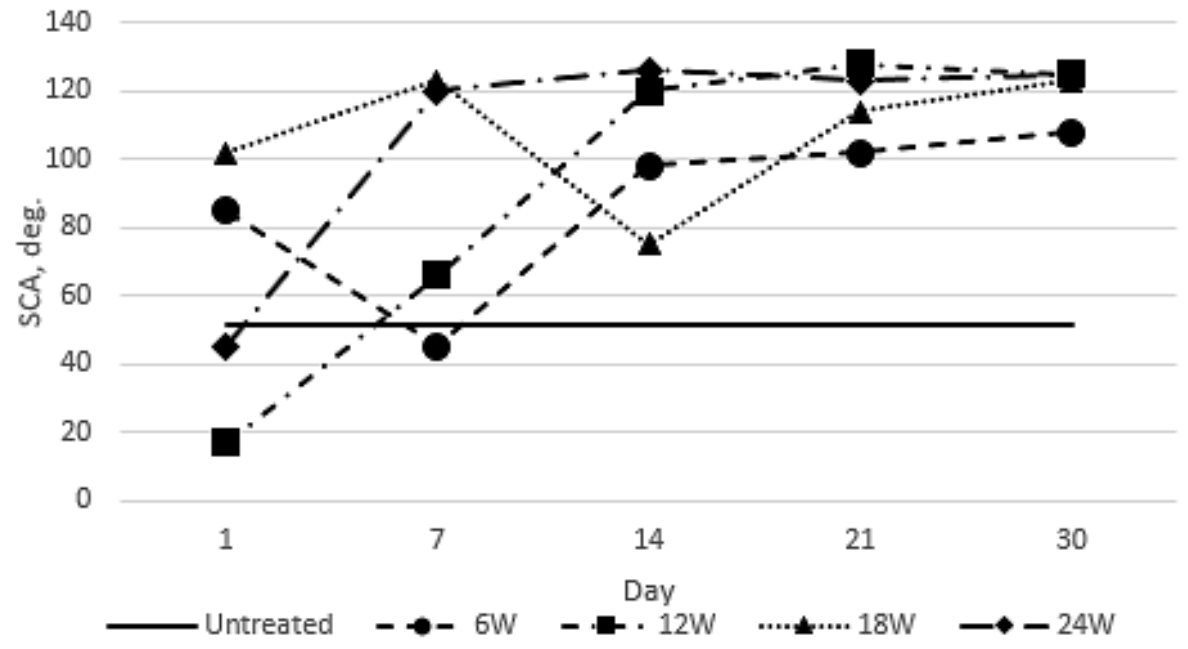

Figure 6. Evolution of wetting and static contact angle measurement with an increment of laser power output

\section{Effect of Surface Profile on Contact Angle}

Cai, et al. [16] has found one dimensionless functional parameter, which helps to assess the surface contact angle, namely $R_{h y}$, which can be computed from the $R_{z}$ average ratio to $R_{s m}$. According to Bhushan, et al. [24], an enormous value $R_{h y}$ can be obtained from a large $R_{z}$ or smaller $R_{s m}$, showing that the surface features should have a significant deep or smaller distance in the horizontal direction (i.e., high density). The results on the calculation of $R_{h y}$ parameters are presented in Figure 6, where it shows that the highest $R_{h y}$ value able to produce a hydrophobicity state.

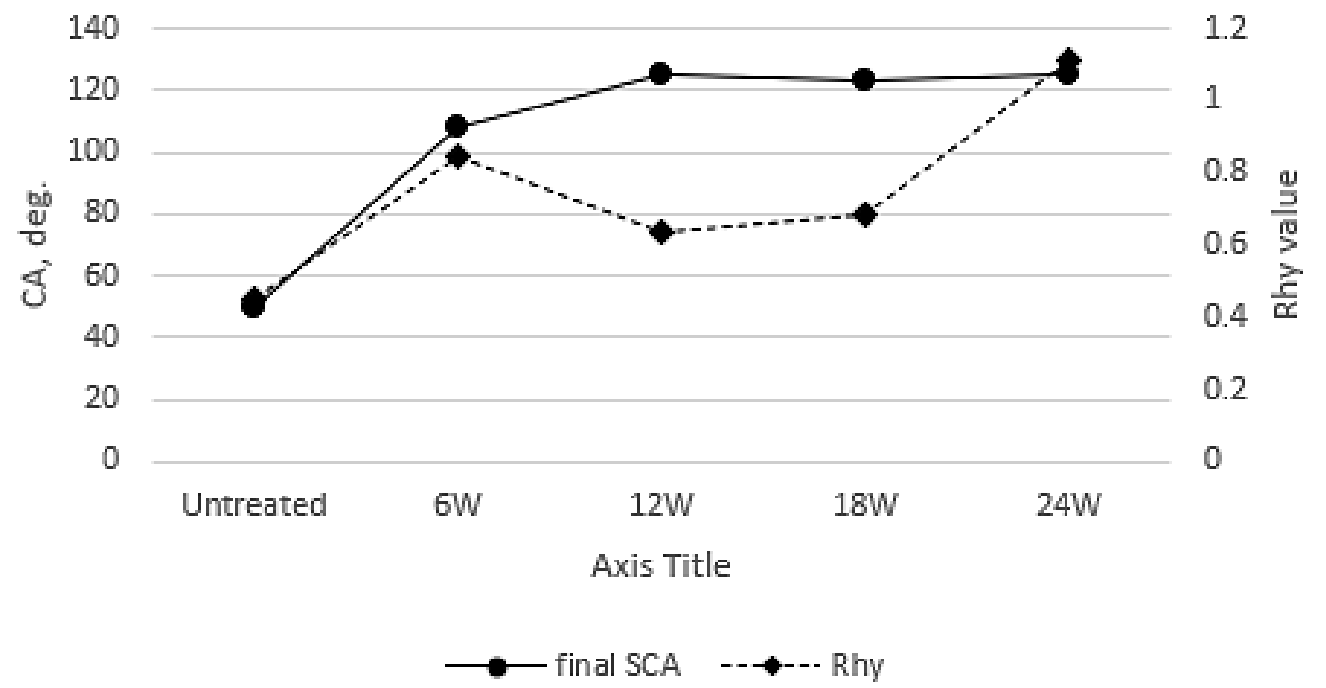

Figure 7. Comparison between $R_{h y}$ value and CA last change (day-30)

Experiments conducted also prove that the surface resulting from $24 \mathrm{~W}$ power output has a lot of air trap area because of the high $R_{s k}$ and $R_{k u}$ value compared to areas with textured surface with 6,12 and $18 \mathrm{~W}$. This air trap's effect will have a specific relation to the surface model of wetting behaviour from Cassie-Baxter, which explains the rough surface and contact angle stability function.

\section{Effect of Surface Profile on Contact Angle}

The wetting model can be explained graphically, as shown in Figure 7. Based on the current Cassie-Baxter model, the droplet cannot access the spaces of the texture morphology. However, to assure that the droplet cannot be attached to the microstructures base, the droplet height between the microstructure should be smaller than the depth of the microstructure. The deep valley also contributes to forming stable air trap (pockets) underwater droplets, which creates superhydrophobic surfaces. Therefore, adequate depth of the microstructure is necessary for the Cassie-Baxter water droplet condition [4, $16,25]$.

The hydrophilic phenomenon after laser texturing, showed by a low contact angle, can be explained primarily by the Wenzel model because of increasing surface roughness [4, 10, 23] as expressed by 


$$
\cos \theta_{w}=r \cos \theta_{f}
$$

Where;

$\theta_{w}$ is the apparent contact angle of the surface after laser texturing;

$\theta_{f}$ is the equilibrium contact angle on the same material flat surface; and,

$r=\frac{\text { actual surface area }}{\text { planar area }}$

$r(>1)$ is the surface roughness factor [18].

As surface roughness increases directly after texturing, the apparent contact angle, $\theta_{w}$, will decrease because of the increase of $r$ based on Eq. 2. Wenzel's model cannot, therefore, clarify the transition over time from hydrophilic to hydrophobic. Therefore, the improvement in weight resistance is attributed to surface chemistry as surface morphology does not change. From Eq. 2, it suggests that surface chemistry, which induces a change in $\theta_{f}\left(\Delta \theta_{f}\right)$, would support a comparative variation in $\theta_{w}\left(\Delta \theta_{w}\right)$ as the following equation:

$$
\Delta \theta_{w}=r\left(\frac{\sin \theta_{f}}{\sin \theta_{w}}\right) \Delta \theta_{f}
$$

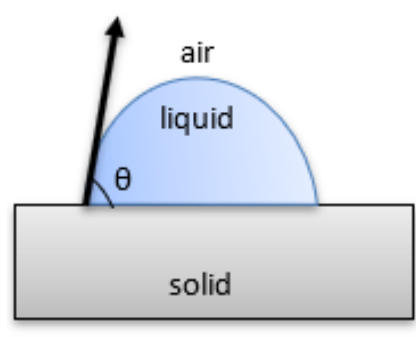

(a)

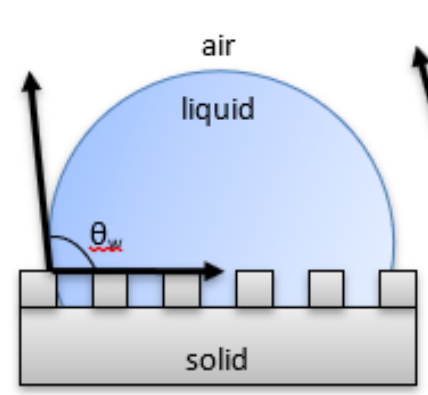

(b)

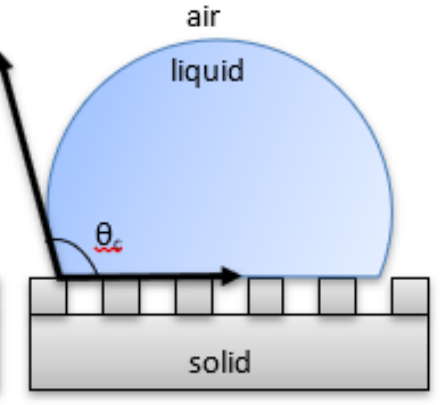

(c)

Figure 8. Wetting behaviour of: (a) Young model, (b) Wenzel model and (c) Cassie model

Both Wenzel's and Cassie-touch Baxter's angles are closely linked to surface topography's vertical and horizontal characteristics [16]. The gaseous phase entrapment will increase the contact angle, as shown in Figure 8, even if the hydrophobic surface is preferably smooth. Besides, it can be concluded that too rough surfaces got in this work with the ns-pulsed laser fibre entanglement do not allow the quick reduction in the angle of contact with water as expressed by Wenzel [4]. The change in the chemical composition of the surface is another significant factor in reducing the contact angle. Such variations can be interpreted as changes to an ideally flat surface's equivalent contact angle. Surface oxidation is also expected during processing in the ambient atmosphere [26].

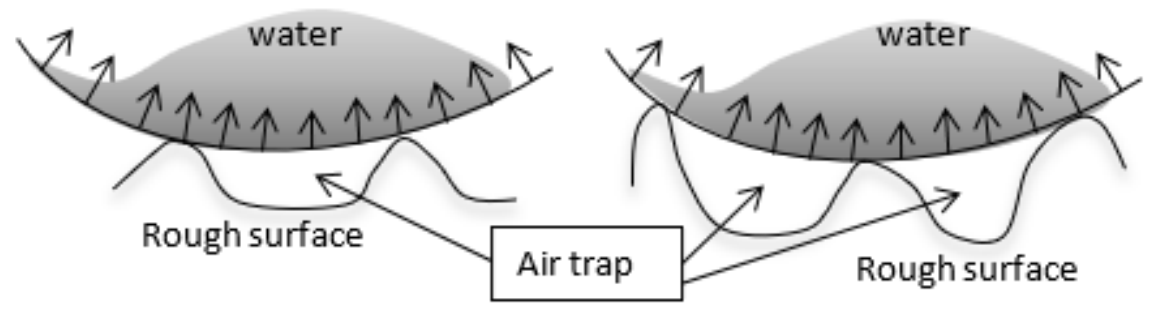

Figure 9. The higher surface profile of $R_{s k}$ and $R_{k u}$ will make more space for air trapped under the water droplet

\section{CONCLUSIONS}

The effect of nanosecond laser parameter of the laser power and the surface roughness $\left(R_{k u}\right.$ and $\left.R_{s k}\right)$ was studied on SS316L. The maximum contact angle of $125^{\circ}$ can be achieved with the output power of $24 \mathrm{~W}$ and calculated on the $30^{\text {th }}$ day. The conclusions that can be reached are: 
i. The laser output power has an enormous influence on the contact angle as the surface roughness increases. The surface topography changes with the various laser output power, and the contact angle will increase before it reaches to the peak value for a certain period of time.

ii. The contact angle will increase with the increase of $R_{s k}$ and $R_{k u}$ under the same microstructural pitch. The high value of $R_{s k}$ and $R_{k u}$ means more space for the droplet to settle. Therefore, the creation of an air trap (pocket) in the water droplet is better and the droplet stable on the specimen surface in the state of Cassie-Baxter.

\section{ACKNOWLEDGMENTS}

The author would like to thank the technical team at Universiti Malaysia Pahang for their work in providing laboratory facilities for materials preparation and laser texturing equipment at the Faculty of Mechanical and Automotive Engineering Technology, including the 3D confocal microscope at the Faculty of Manufacturing Engineering. Financial funding for research grants RDU1803171 and PGRS200302 by the Ministry of Higher Education through Universiti Malaysia Pahang are also appreciated.

\section{REFERENCES}

[1] A. Dunn et al., "Laser surface texturing for high friction contacts," Applied Surface Science, vol. 357, pp. 2313-2319, 2015.

[2] E. Hernandez, M. Alfano, G. Lubineau, and U. Buttner, "Improving adhesion of copper/epoxy joints by pulsed laser ablation," International Journal of Adhesion and Adhesives, vol. 64, pp. 23-32, 2016.

[3] G. Hu, K. Guan, L. Lu, J. Zhang, N. Lu, and Y. Guan, "Engineered functional surfaces by laser microprocessing for biomedical applications," Engineering, vol. 4, no. 6, pp. 822-830, 2018.

[4] Y. Shi, Z. Jiang, J. Cao, and K. F. Ehmann, "Texturing of metallic surfaces for superhydrophobicity by water jet guided laser micro-machining," Applied Surface Science, vol. 500, p. 144286, 2020.

[5] A. Sulaiman, M. Aiman, M. Quazi, M. Ishak, and T. Ariga, "Enhancement of mechanical properties of Copper Brazed by laser surface modification," in IOP Conference Series: Materials Science and Engineering, 2020, vol. 788, no. 1: IOP Publishing, p. 012012.

[6] K. Yaakob, M. Ishak, and S. Idris, "The effect of pulse welding parameters on weld geometry of boron steel using low power fibre laser," Journal of Mechanical Engineering and Sciences, vol. 11, no. 3, pp. 2895-2905, 2017.

[7] A. Zaifuddin, M. Aiman, M. Quazi, M. Ishak, and T. Ariga, "Effect of laser surface modification (LSM) on laser energy absorption for laser brazing," in IOP Conference Series: Materials Science and Engineering, 2020, vol. 788, no. 1: IOP Publishing, p. 012013.

[8] S. F. Roduan et al., "Effect of LST parameter on surface morphology of modified copper substrates," Jurnal Tribologi, vol. 26, pp. 84-91, 2020.

[9] C. Sun, J. Min, J. Lin, H. Wan, S. Yang, and S. Wang, "The effect of laser ablation treatment on the chemistry, morphology and bonding strength of CFRP joints," International Journal of Adhesion and Adhesives, vol. 84, pp. 325-334, 2018.

[10] Y. Wang, C. Ke, T. Wu, X. Zhao, and R. Wang, "Nanosecond laser texturing with hexagonal honeycomb micro-structure on Titanium for improved wettability and optical properties," Optik, vol. 192, p. 162953, 2019.

[11] S. Li et al., "Biomimetic robust superhydrophobic stainless-steel surfaces with antimicrobial activity and molecular dynamics simulation," Chemical Engineering Journal, vol. 372, pp. 852-861, 2019.

[12] K. Sun et al., "Anti-biofouling superhydrophobic surface fabricated by picosecond laser texturing of stainless steel," Applied Surface Science, vol. 436, pp. 263-267, 2018.

[13] M. Rafieazad, J. Jaffer, C. Cui, X. Duan, and A. Nasiri, "Nanosecond laser fabrication of hydrophobic stainless steel surfaces: The impact on microstructure and corrosion resistance," Materials, vol. 11, no. 9, p. 1577, 2018.

[14] M. Conradi, T. Sever, P. Gregorčič, and A. Kocijan, "Short-and long-term wettability evolution and corrosion resistance of uncoated and polymer-coated laser-textured steel surface," Coatings, vol. 9, no. 9, p. 592, 2019.

[15] G. Hu, Y. Song, and Y. Guan, "Tailoring metallic surface properties induced by laser surface processing for industrial applications," Nanotechnology and Precision Engineering, vol. 2, no. 1, pp. 29-34, 2019.

[16] Y. Cai, X. Luo, Z. Liu, Y. Qin, W. Chang, and Y. Sun, "Product and process fingerprint for nanosecond pulsed laser ablated superhydrophobic surface," Micromachines, vol. 10, no. 3, p. 177, 2019.

[17] F. Fraggelakis, G. Mincuzzi, J. Lopez, I. Manek-Hönninger, and R. Kling, "Texturing metal surface with MHz ultra-short laser pulses," Optics Express, vol. 25, no. 15, pp. 18131-18139, 2017.

[18] J. Li, Y. Zhou, F. Fan, F. Du, and H. Yu, "Controlling surface wettability and adhesive properties by laser marking approach," Optics \& Laser Technology, vol. 115, pp. 160-165, 2019.

[19] C. V. Ngo and D. M. Chun, "Effect of Heat Treatment Temperature on the Wettability Transition from Hydrophilic to Superhydrophobic on Laser-Ablated Metallic Surfaces," Advanced Engineering Materials, vol. 20, no. 7, p. 1701086, 2018.

[20] C.-V. Ngo and D.-M. Chun, "Fast wettability transition from hydrophilic to superhydrophobic laser-textured stainless steel surfaces under low-temperature annealing," Applied Surface Science, vol. 409, pp. 232-240, 2017. 
[21] C. L. Donaghy et al., "Fibre laser treatment of beta TNZT titanium alloys for load-bearing implant applications: Effects of surface physical and chemical features on mesenchymal stem cell response and Staphylococcus aureus bacterial attachment," Coatings, vol. 9, no. 3, p. 186, 2019.

[22] V. D. Ta et al., "Laser textured superhydrophobic surfaces and their applications for homogeneous spot deposition," Applied Surface Science, vol. 365, pp. 153-159, 2016/03/01/ 2016.

[23] A. Dunn et al., "Laser textured surface gradients," Applied Surface Science, vol. 371, pp. 583-589, 2016.

[24] B. Bhushan, M. Nosonovsky, and Y. Chae Jung, "Towards optimization of patterned superhydrophobic surfaces," Journal of the Royal Society Interface, vol. 4, no. 15, pp. 643-648, 2007.

[25] Y. Cai, W. Chang, X. Luo, A. M. Sousa, K. H. A. Lau, and Y. Qin, "Superhydrophobic structures on 316L stainless steel surfaces machined by nanosecond pulsed laser," Precision Engineering, vol. 52, pp. 266-275, 2018.

[26] G. Menci, A. G. Demir, D. G. Waugh, J. Lawrence, and B. Previtali, "Laser surface texturing of $\beta$-Ti alloy for orthopaedics: Effect of different wavelengths and pulse durations," Applied Surface Science, vol. 489, pp. 175-186, 2019. 